\section{Some notes on the entropic theory of perception (Reply to A. W. MacRae)}

\author{
KENNETH H. NORWICH \\ University of Toronto, Toronto, Canada
}

My paper ("The Magical Number Seven: Making a 'Bit' of 'Sense," ' Norwich, 1981a) was preceded by two others written by Professor J. C. Baird of Dartmouth College (Baird, 1970a, 1970b), in which Baird sought, honestly and skillfully, to introduce an information theoretical base into psychophysics. A. W. MacRae followed (1972) with a blistering attack on Baird's work-an eight-page diatribe of destructive criticism. Baird was not granted the privilege of a rejoinder.

My 1981 paper again seeks an explanation for psychophysical phenomena within information theory (an entropic theory of perception), and MacRae is again on the attack. His comments are not profound. The core of his objection, as we shall see below, seems to derive from a difficulty with the arithmetic.

Let us proceed through MacRae's (1982) commentary.

MacRae, page 593: "Equation 8 is described as a definition of $H_{s} \ldots$ not necessarily consistent with ... the definition for the same symbol given on page 410 "

On page 410 (Norwich, 1981a), $\mathrm{H}_{\mathrm{s}}$ is defined for a Garner-Hake experiment. In such an experiment, human subjects talk and report their judgments. In Equation $8, \mathrm{H}_{\mathrm{s}}$ is defined for a dissected, isolated sensory receptor and neuron. Dissected preparations of frog peroneal nerve and cat's auditory nerve do not usually talk. But we can measure their electrical activity. Therefore, a second definition of $\mathrm{H}_{\mathrm{s}}$ in terms of electrical activity was necessary. Perhaps MacRae knows how to converse with such preparations. He could then merge the two definitions of $\mathrm{H}_{\mathrm{s}}$. There is absolutely no lack of consistency.

MacRae, page 591: "The 'Psychophysical Adaptation Curve" "

This concept introduces the entropy function, $\mathrm{H}_{\mathrm{s}}$. It is a convenience only. The paper was submitted originally without this section. Reviewers found it easier to conceive the meaning of $\mathrm{H}_{\mathrm{s}}$ when the psychophysical adaptation curve was considered first. If

The author is affiliated with the Department of Physiology and the Institute of Biomedical Engineering, University of Toronto, Toronto, Ontario MSS 1A4, Canada.
MacRae does not like it, he can disregard it. The remainder of the paper is totally independent of the concept of the psychophysical adaptation curve, and can be read perfectly well without it.

MacRae, page 592: 'But what Norwich turns out to be interested in estimating is the ratio of the maximum to the minimum firing rate"

Dead wrong. MacRae seems to have difficulty in distinguishing a minus from a division sign. Equation 7 states clearly

$$
k I_{t \max }=F_{\max }-F_{\min } \text {. }
$$

The difference between firing rates is relatively insensitive to the exact values of the firing rate recorded experimentally. Regardless of how the maximum and minimum values are estimated from the experimental curves, the estimated values of the information transmitted will not likely deviate by more than $10 \%$ or $15 \%$ (see example below).

MacRae, page 592: "Thus, Norwich offers three conflicting interpretations of the relationship between firing rate and time""

No. Only one interpretation is offered, and it is discussed in some detail by Norwich (Note 1). Some receptors adapt incompletely (e.g., stretch); some adapt completely (e.g., taste). Each receptor must have a maximum "local" memory span which, depending on the kind of receptor, may be several hundred milliseconds to about $2 \mathrm{~min}$. Call this maximum memory span $\tau_{\max }$. Then $\tau$ in Equation $15^{1}$ must always be equal to or less than $\tau_{\max }$. The receptor cannot, then, "remember" stimuli which occurred longer ago than $\tau_{\max }$. This seems patently obvious, since, for example, the fibers in the auditory nerve are not firing now in response to sounds heard half an hour ago. When $\tau=\tau_{\max }$ is introduced into Equation $15, F$ will not always be vanishingly close to zero. Hence, the plateau in firing rate.

But if MacRae demands slavish obedience to Equations 7 and 15, let's grant it to him. For the acoustic case in Table 2 of Norwich (1981a), we take $F_{\max }$ equal to 320 impulses/sec, which is its measured value (no "intuitively based ad hoc adjustment[s]," which MacRae charges on page 592), and $F_{\min }=0$ (no plateaus which "do not follow," as MacRae charges on page 592). Then the transmitted information equals $(320-0) / 83.39=3.837$ bits. The result reported in Table 2 of Norwich (1981a) is 3.84 bits. The results in this case are identical. MacRae seems incapable of understanding that small changes in 
quantities that are subtracted produce small changes in their difference.

MacRae, pages 592-593: 'Signal, Noise, and Information"

MacRae's problem seems to center around the meaning of the term "noise" as it is used in this context.

The variance of a population of signals is taken to be $\sigma^{2}$. The variance of the means of samples of this population of size $\mathrm{n}$ is then $\sigma^{2} / \mathrm{n}$. The quantity $\sigma / \sqrt{\mathrm{n}}$ is familiar to most behavioral scientists as the standard error of the mean. As the sample size, $n$, increases, $\sigma / \sqrt{\mathrm{n}}$ decreases, and there is an increase in our confidence that the sample mean approaches the population mean. Expressed another way, as the sample size, $n$, increases, our uncertainty about the population mean decreases. (Basically, this is what the whole paper is about: the longer a steady stimulus is observed, the more certain we are about its characteristics.)

How, then, is the quantity $\sigma^{2} / \mathrm{n}$ appraised by a sensory receptor? Unfortunately, we do not know exactly. But it is not likely a digital calculation such as one would perform with a hand calculator. Any measure by the receptor of a central tendency in the signal is probably reached by analog computation (e.g., by summing electrical signals), and analog computations are always limited by the presence of noise. The $\mathrm{N}^{2}$ that appears in Equation $\mathbf{9}$ and thereafter is a measure of this noise which limits the resolution of the estimate of the mean made by the receptor circuitry. The $\mathrm{H}_{\mathrm{s}}$ function in Equation 9 is a model of the progressive increase in certainty attending the continued observation of a steady signal. As stated in the final two lines of Appendix B of Norwich (1981a), "Only time and testing will determine the utility of the function."

It is interesting to consider the case in which, instead of observing the magnitude of a tone or a force, we observe the position and momentum of a small particle (Norwich, 1981b). The probability functions, $P_{S N}$, are then the wave functions of the particle. It can then be shown, using Equation 8 (Norwich, 1981a) for gaussian wave functions, that the product of the uncertainties in position and momentum of the particle must always be equal to or exceed the value of $\mathrm{N}^{2}$. Again, $\mathrm{N}^{2}$ limits the resolution of perception. That is, we can derive from a psychophysical base the uncertainty principle of Heisenberg, where $h^{2}$ is a measure of $\mathrm{N}^{2}$ ( $h$ is Planck's constant).

But, if the proof of a pudding is in the eating, then the proof of a theory is in its predictions. One of the predictions issuing from the entropic interpretation of adaptation curves can easily be tested by readers of this journal. Adaptation curves giving the decline in mean or median magnitude estimates with increasing time of stimulus application have been measured by many psychophysicists. For example, perceived brightness or perceived intensity of taste diminish with the stimulus duration. There is good reason to expect that changes in perceived intensity of a stimulus parallel changes in the electrical activity of the associated sensory neurons (e.g., Borg et al., 1970). Therefore, F in Equation 15 (Norwich, 1981a) may be measured approximately by magnitude estimates of stimulus intensities (but not necessarily line length or two-point discrimination). By means of the fairly straightforward curve-fitting process shown in Appendix $A$, and one simple calculation, as shown in Tables 1 and 2, the channel capacities (bits per stimulus) can be estimated from the adaptation curves. Many good compendiums of channel capacity are available to check the results (e.g., Baird, 1970a). Therefore, readers with access to such adaptation data can check, with a minimum of labor, the approximate truth of Equation 15.

\section{REFERENCE NOTE}

1. Norwich, K. H. On the relationship between physics and physiology (Report No. 18, Institute of Biomedical Engineering). Toronto: University of Toronto, 1978.

\section{REFERENCES}

BAIRD, J. C. A cognitive theory of psychophysics. I. Information transmission, partitioning, and Weber's law. Scandinavian Journal of Psychology, 1970, 11, 35-46. (a)

BAIRD, J. C. A cognitive theory of psychophysics. II. Fechner's law and Stevens' law. Scandinavian Journal of Psychology, 1970, 11, 89-102. (b)

Borg, G., Diamant, H., \& Zotterman, Y. Neural and perceptual responses to taste stimuli. In G. E. W. Wolstenholme \& J. Knight (Eds.), Taste and smell in vertebrates. London: Churchill, 1970.

MacRAe, A. W. Information transmission, partitioning and Weber's law. Some comments on Baird's cognitive theory of psychophysics. Scandinavian Journal of Psychology, 1972, 13, 73-80.

MacRae, A. W. The magical number fourteen: Making a very great deal of non-sense. Perception \& Psychophysics, 1982, 31, 591-593.

Norwich, K. H. The magical number seven: Making a "bit" of "sense." Perception \& Psychophysics, 1981, 29, 409-422. (a)

Norwich, K. H. Uncertainty in physiology and physics. Bulletin of Mathematical Biology, 1981, 43, 141-149. (b)

\section{NOTE}

1. All equation numbers refer to Norwich, $1981 \mathrm{a}$.

(Manuscript received March 8, 1982; accepted for publication March 12, 1982.) 